\title{
An image-enhancement method based on variable-order fractional differential operators
}

\author{
Mengjia Xu ${ }^{\mathrm{a}, \mathrm{b}}$, Jinzhu Yang ${ }^{\mathrm{a},{ }^{*}}$, Dazhe Zhao a and Hong Zhao ${ }^{\mathrm{a}}$ \\ ${ }^{a}$ Key Laboratory of Medical Image Computing of Ministry of Education, Northeastern University, \\ Shenyang, Liaoning 110819, China \\ ${ }^{b}$ Division of Applied Mathematics, Brown University, Providence, RI 02912, USA
}

\begin{abstract}
In this study, we develop a new algorithm based on fractional operators of variable-order in order to enhance image quality. First, three kinds of popular high-order discrete formulas are adopted to obtain the coefficients, and subsequently, a mask optimization method for selecting the fractional order adaptively is applied to construct a variable-order fractional differential mask along with the coefficients generated from the first step. We carry out experiments on OCT thoracic aorta images and some nature images with low contrast and noise, demonstrating that the high-order discrete method leads to significantly better performance in enhancing the edge information nonlinearly compared to the standard first-order discrete method. Moreover, the optimized mask with variable-order of the fractional derivative not only can preserve the edge information of the processed images adequately, but it also effectively suppresses the noise in the smooth area.
\end{abstract}

Keywords: Image enhancement, fractional differential operator, variable-order, mask optimization

\section{Introduction}

Since the OCT (Optical Coherence Tomography) technology can provide noninvasive, in vivo, three dimensional cross-sectional imaging of tissue microstructure, it has been widely used in the diagnosis of retinal and intra-arterial disease (e.g. thoracic aortic aneurysm) applications [1, 2]. However, the quality of OCT images is usually affected by speckle noise introduced in the imaging procedure, which degrades the contrast by obscuring small and low intensity features [3]. Therefore, speckle noise blurs the boundaries between different tissues, and thus seriously reduces the accuracy of image segmentation and pattern recognition in OCT image.

To overcome the disturbance of speckle noise and obtain a good quality of OCT image is still an extremely challenging problem in the image processing field. Previously, a number of image enhancement methods for OCT images have been proposed. These methods can be broadly classified into three types: machine learning-based filtering method, PDE-based linear or nonlinear anisotropic

\footnotetext{
* Address for correspondence: Jinzhu Yang, Key Laboratory of Medical Image Computing of Ministry of Education, Northeastern University, Shenyang, Liaoning 110819, China. Tel.: +86 13889386965; Fax: +86 83663446; E-mail: yangjinzhu@ise.neu.edu.cn.
}

0959-2989/15/\$35.00 @ 2015 - IOS Press and the authors. 
diffusion method, and fractional differential operator based method. Mohammad, et al. [4] developed a segment-wise image de-noising method that adopted the Artificial Neural Network (ANN) method to estimate the noise parameters and sigma of Rayleigh distribution for the modeling of noise in the image, then generated the noise model image through solving the inverse Rayleigh function by numerical method. However, this method is prone to being influenced by training sample data and has a high computational complexity. To our knowledge, the non-linear anisotropic diffusion method has been applied to MRI image enhancement as early as 1992, but more recently, Harry, et al. [5] first developed a PDE-based complex nonlinear diffusion method for OCT image enhancement, which introduced a directional diffusion coefficient (phase angle) to remove speckle noise from the edges while preserving the structure similarity without the "staircasing effect". Despite this progress, none of the aforementioned methods can capture the non-linear, non-causal, non-Gaussian, non-even and nonintegral differential features of the speckle noise.

Therefore, to better deal with the above OCT image enhancement problem, fractional-order PDEs based modeling method has been looked as a potentially powerful and effective tool for solving these problems in recent years. In earlier work, Marko, et al. [6] presented a fully fractional anisotropic diffusion equation for noise removal combined with both spatial and temporal fractional derivatives. It performs better than the baseline anisotropic diffusion methods. Hesthaven, et al. [7] developed an algorithm based on the fractional Laplacian operator approximated by $h p$-finite element numerical method, which can enhance the noisy image in different portions independently. On the base of two commonly used definitions for fractional differential (Grünwald-Letnikov and Riemann-Liouville), $\mathrm{Pu}$, et al. [8] developed the so-called YIFEIPU-1 algorithm that adopted the coefficients of factional differential derivative to generate five different kinds of masks for image filtering, but their approach suffers from distortion while dealing with color images in the RGB space. $\mathrm{Yu}$, et al. [9] improved the original YIFEIPU-1 method on texture enhancement by using a second-order Riesz fractional differential operator, with obvious improvements both for grayscale image enhancement as well as color image enhancement. However, how to adapt the fractional order according to the complexity of features in different portions of the image is a big challenge at present. To this end, $\mathrm{Hu}$, et al. [10] adopted a cross-based local support region construction approach to accomplish adaptive image texture enhancement with a non-integer fractional differential step and order. However, the discretization error is usually ignored in this method, which will inevitably influence the image enhancement results to a great extent as well.

This paper will mainly focus on different discrete formulas for the coefficients of mask and adaptive fractional derivative order selection method for constructing the mask, and is organized as follows. Section 2 firstly recalls on some basic theoretical background of fractional differential and derivative, then three different types of discrete formulas are given to generate the coefficients of mask, and a mask optimization process is implemented to construct a variable-order fractional mask. Results from our experiments and analysis are presented in Section 3. Finally, we summarize the work in Section 4.

\section{Image enhancement based on variable-order fractional differential operators}

In this section, a new image enhancement method based on variable-order fractional differential operators is presented. As shown in Figure 1, the proposed method contains four main steps: 1) Fractional derivative discretization, 2) Fractional mask construction, 3) Mask optimization and 4) Convolution with original image. Once the discretization coefficients $C_{m}^{v}, v \in(0,1)$ of fractional 


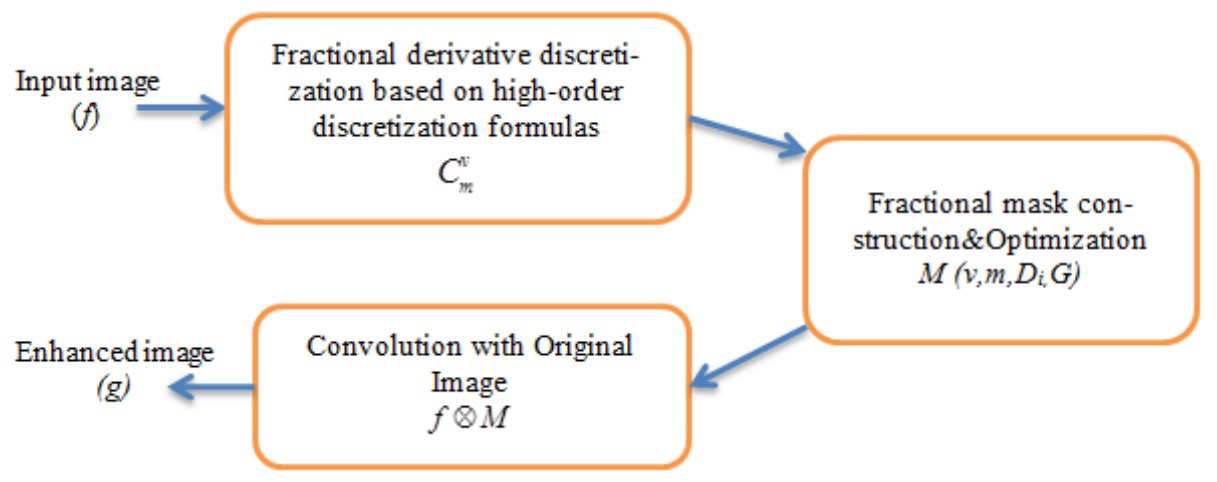

Fig. 1. Flow chart of the proposed method.

derivatives are obtained by adopting discretization formulas $\left\{D_{i}\right\}$, where $\mathrm{i}=1,2,3,4,5$, a fractional differential mask (or operator) $M\left(v, m, D_{i}, G\right)$ of size $\boldsymbol{m}$ can be constructed. In addition, a gradient $(G)$ based mask optimization function is used to optimize the order $v$ of the fractional differential derivative adaptively, which results in different values of $v$ and $C_{m}^{v}$ in each direction of the masks eventually.

\subsection{Fractional derivative definition}

The fractional integral (or the Riemann-Liouville integral) [11] with order $v>0$ of the given function $f(t)$ is defined as Eq. (1).

$$
{ }_{a} D_{x}^{-v} f(x)=\frac{1}{\Gamma(v)} \int_{a}^{x}(x-s)^{v-1} f(s) d s
$$

where $\Gamma($.$) is Euler's gamma function. Following this the definition of left and right Riemann-$ Liouville (RL) derivatives with order $n-1<v<n$ of a given function $f(x)$ in a finite domain $[a, b]$ are given by Eqs. (2) and (3), respectively:

$$
\begin{aligned}
& { }_{a} D_{x}^{v} f(x)=\frac{d^{n}}{d x^{n}}\left[{ }_{a} D_{x}^{-(n-v)} f(x)\right]=\frac{1}{\Gamma(n-v)} \frac{d^{n}}{d x^{n}} \int_{a}^{x}(x-s)^{n-v-1} f(s) d s \\
& { }_{x} D_{b}^{v} f(x)=\frac{d^{n}}{d x^{n}}\left[{ }_{x} D_{b}^{-(n-v)} f(x)\right]=\frac{(-1)^{n}}{\Gamma(n-v)} \frac{d^{n}}{d x^{n}} \int_{x}^{b}(s-x)^{n-v-1} f(s) d s
\end{aligned}
$$

Note that when $v=n$, the fractional derivative is equivalent to an integer-order differential derivative.

\subsection{Coefficient generation based on different discrete formulas}


For the discrete characteristic of digital images, when the fractional differential derivative in Eq. (2) above is applied in image processing, it should be transformed into discrete format. The discretization of the R-L fractional differential derivative at the point of $x=x_{j}$ can be generalized as Eq. (4).

$$
\left[{ }_{a} D_{x}^{v} f(x)\right]_{x=x_{j}}=\frac{1}{h^{v}} \sum_{j=0}^{n} C_{j}^{v} f\left(x_{n-j}\right)+\mathrm{O}\left(h^{p}\right)
$$

where $h$ is the step size, $v$ is the order of fractional differential derivative, $C_{j}^{v}$ is the coefficient that determines the discretization order $p$ of the R-L fractional operator. In existing fractional differential based image enhancement approaches, the selection of discretization method is usually ignored; however, different discretization formulas will generate different coefficients for the mask, which will have an influence on the performance of image enhancement as well. Most of the existing fractional mask based image enhancement methods rarely adopt high-order discretization formulas for the coefficients generation of the mask. Therefore, three different types of high-order discretization formulas are given below for the generation of coefficients that are used for the construction of convolution masks in section 2.3.

\subsubsection{Case 1: Fractional backward difference formula of order $p$ (FBDF-p)}

For the discretization scheme FBDF with order of $p$, the coefficients $C_{j}^{v}$ in Eq. (4) can be obtained by a recurrence equation [12] as Eq. (5).

$$
C_{j}^{v}=\frac{1}{j u_{0}} \sum_{i=0}^{j-1}[v(j-i)-i] C_{i}^{v} u_{j-i}
$$

where $u_{j}$ satisfies the following equation

$$
\sum_{j=1}^{p} \frac{1}{j}(1-z)^{j}=\sum_{j=1}^{p} u_{j} z^{j}
$$

\subsubsection{Case 2: Weighted (shifted) Grunwald-Letnikov formula I (WG-I)}

For the second-order WSG-I, the coefficients $C_{j}^{v}$ in Eq. (4) satisfies [13]

$$
C_{0}^{v}=\frac{v}{2} \omega_{0}^{v}, C_{j}^{v}=\frac{v}{2} \omega_{j}^{v}+\frac{2-v}{2} \omega_{j-1}^{v}, j \geq 1
$$

where $\omega_{j}^{v}=\frac{1}{\Gamma(-v)} \frac{\Gamma(j-v)}{\Gamma(j+1)}$

\subsubsection{Case 3: Weighted (shifted) Grunwald-Letnikov formula II (WG-II)}


For the second-order WSG-II, the coefficients $C_{j}^{v}$ in Eq. (4) satisfies [13]

$$
C_{0}^{v}=\frac{v+2}{4} \omega_{0}^{v}, C_{1}^{v}=\frac{v+2}{4} \omega_{1}^{v}, C_{j}^{v}=\frac{v+2}{4} \omega_{j}^{v}+\frac{2-v}{4} \omega_{j-2}^{v}, j \geq 2
$$

where $\omega_{0}^{v}=1, \omega_{j}^{v}=\left(1-\frac{v+1}{j}\right) \omega_{j-1}^{v}, j=1,2, \ldots$.

\subsection{Fractional mask construction and optimization}

Similar to the construction of integer-order differential operators, for a fractional differential operator construction, the coefficients generated from the above three different types of discretization formulas are used here. The fractional mask with a size of $(2 m+1) \times(2 m+1)$ can be defined as follows:

Note that for computing convenience, all the elements in the fractional mask matrix are normalized by dividing by the term $\operatorname{Sum}=\sum_{j=0}^{m} C_{j}^{v}(j=0,1, \ldots, m)$ to make the sum of elements in the new mask equal to 1 . In addition, the 8 symmetric directions in the mask with same structures lead to an antidirectional capability, which can enhance the image from different directions simultaneously, thus it can efficiently avoid the disadvantage of standard first-order differential operators (e.g. Sobel, Robert, Prewitte, etc.) that can only enhance the boundaries of image in specific directions. It can be observed that the orders of fractional derivative in all 8 directions are completely equal in Figure 2. However, owing to the frequency response of a fractional differential operator, which can be viewed as a nonlinear filter in image processing, the high-pass ability is stronger as the order of the fractional derivative $v$ increases. In other words, the noise will be also enhanced along with the edges enhanced in the image when the order of fractional derivative $v$ is large. Thus it is better to obtain a good quality of image by means of applying different orders of fractional derivative to enhance the smooth area in low frequency regions and sharp area in high frequency like edge or noise, so as to preserve the details of image without loss of important edge information, as well as suppressing the noise within the

\begin{tabular}{|c|c|c|c|c|c|c|}
\hline$C_{m}{ }^{v}$ & & & $C_{m}{ }^{v}$ & & & $C_{m}{ }^{p}$ \\
\hline & $\ldots$ & & $\vdots$ & & $\ldots$ & \\
\hline & & $C_{1}{ }^{v}$ & $C_{1}{ }^{v}$ & $C_{1}{ }^{v}$ & & \\
\hline$C_{m}{ }^{v}$ & $\ldots$ & $C_{1}{ }^{v}$ & $8 C_{0}{ }^{v}$ & $C_{1}{ }^{v}$ & $\ldots$ & $C_{m}{ }^{v}$ \\
\hline & & $C_{1}{ }^{v}$ & $C_{1}{ }^{v}$ & $C_{1}{ }^{v}$ & & \\
\hline & $\ldots$ & & $\vdots$ & & $\ldots$ & \\
\hline$C_{m}{ }^{v}$ & & & $C_{m}{ }^{v}$ & & & $C_{m}{ }^{v}$ \\
\hline
\end{tabular}

Fig. 2. Fractional differential mask construction in 8 directions. 
smooth area simultaneously. Thus, to deal with this problem, a gradient-adaptive indicator function is applied to optimize the mask for adjusting the order of fractional derivative in 8 different directions.

\section{Experimental results and analysis}

In this section, some experiements and comparisons for the proposed method are carried out on OCT images and nature image from the open database "MIT-Adobe FiveK Dataset [14]". Figure 3 shows a comparison result of image enhacement by using 5 different kinds of discrete formulas with $5 * 5$ mask. It can be observed from Figure 3 that the FBDF-p discrete method can provide much more clear details in the enhanced image, followed by the WG-I and WG-II method in sequence. Figure 4 gives the average gradient and constrast comparisons for the image quality evaluation of the image enhaned by 5 discrete methods respectively. This provides evidence that the FBDF-p discrete method has a better performance in preserving the details and edge information than the other two secondorder discrete methods (WG-I and WG-II). However, it can be found that the smooth area is enhanced excessively while the edge is enhanced clearly in the plot (c) of Figure 3. Thus, mask optimization is used to solve this problem. The optimized variable-order mask and uniform-order mask are shown, respectively, in Figure 5.

When the optimized mask is applied to implement OCT image enhancement, the result is shown in Figure 6(d). The optimized mask can enhance the outer wall and lumen boundary of the vessel, but without enhancing the speckle noise in the background.

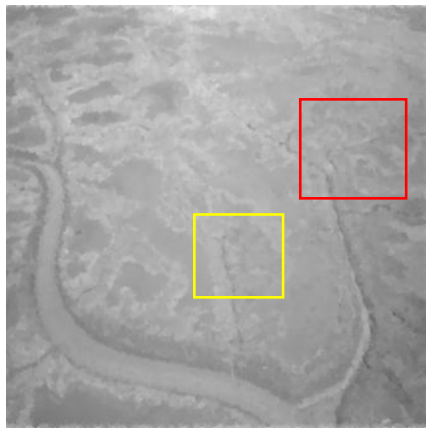

(a) Original image

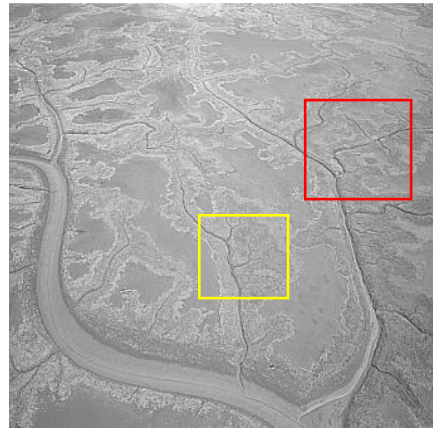

(d) FPDF-3 by $v=0.5$

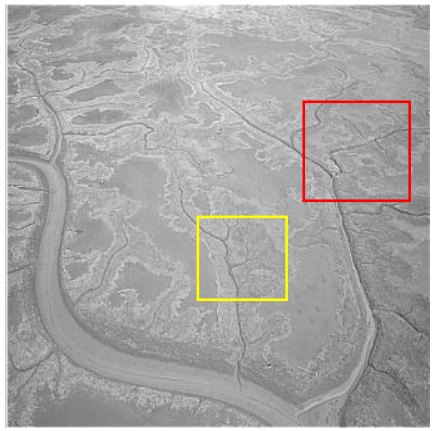

(b) FPDF-1by $v=0.6$

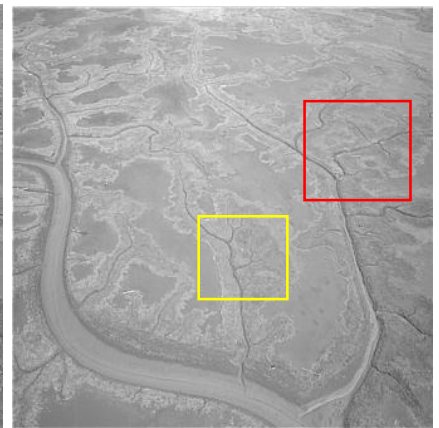

(e) WG-I by $v=0.6$

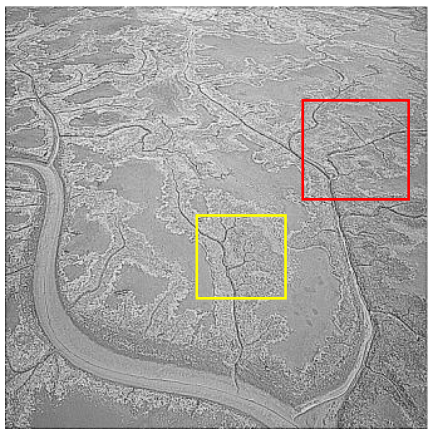

(c) FPDF-2 by $v=0.8$

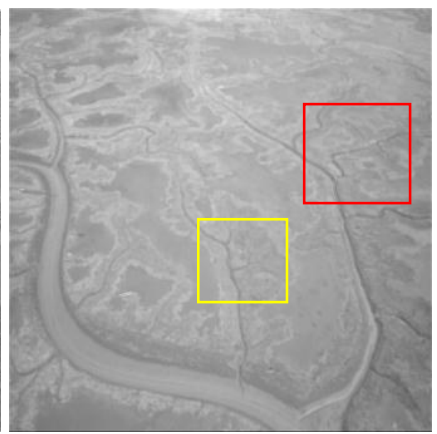

(f) WG-II by $v=0.7$

Fig. 3. Image enhancement results based on FBDF-p $(p=1,2,3)$, WG-II, WG-I method with different order of fractional derivative without mask optimization. 

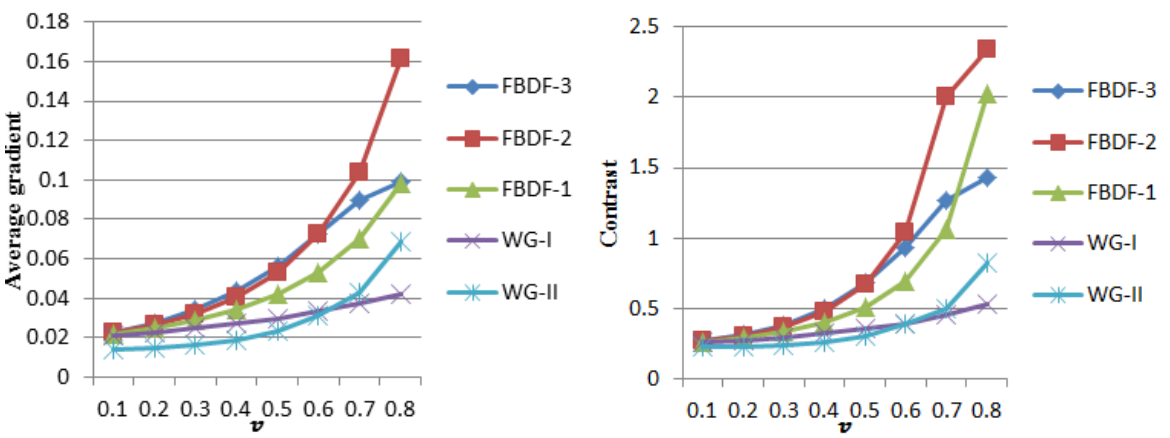

Fig. 4. Average gradient and Contrast results based on five different discrete formulas.

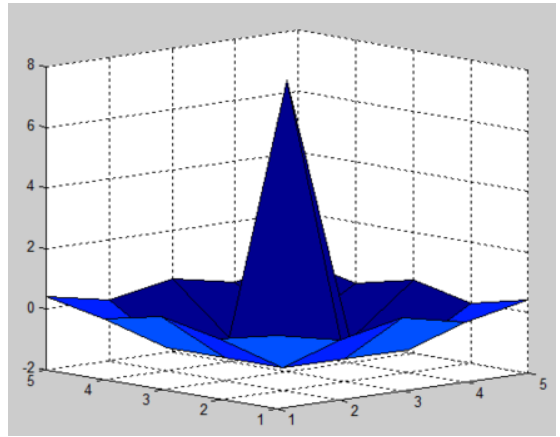

(a) mask without optimization

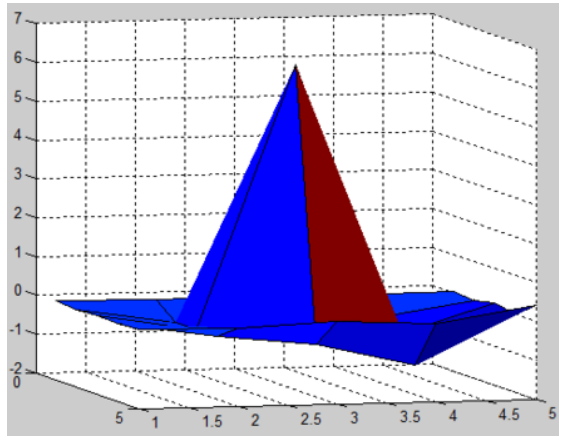

(b) mask after optimization

Fig. 5. Fractional mask constructed by uniform order $(v=0.7)$ and variable-order fractional order.

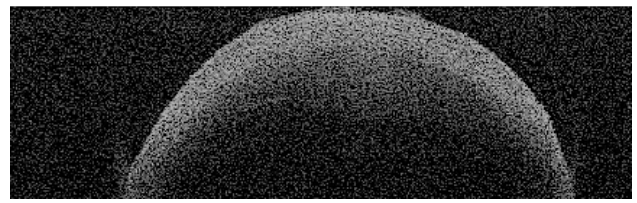

(a) original image

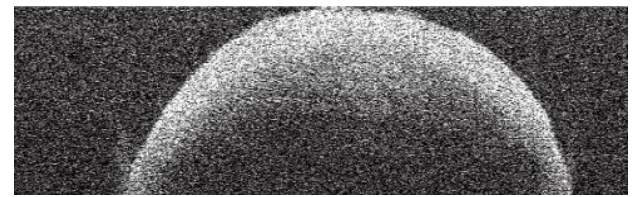

(c) FPDF-2 wih $v=0.8$

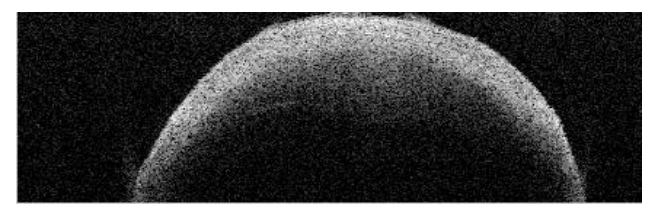

(b) FPDF-2 wih $v=0.4$

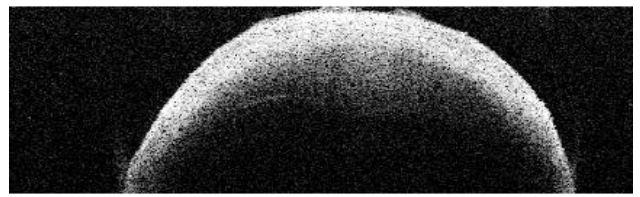

(d) FPDF-2 wih variable-order mask

Fig. 6. OCT image enhancement with optimized mask based on FBDF-3 with $5 * 5$ mask.

Table 1

PSNR comparison results

\begin{tabular}{lllll}
\hline $\begin{array}{l}\text { Noise standard } \\
\text { variance } \sigma\end{array}$ & Tang's method [14] & $\begin{array}{l}\text { FCD-1 } \\
\text { Method [9] }\end{array}$ & Tian's method [13] & Our methods \\
\hline 25 & 28.1793 & 29.5032 & 31.6094 & 34.1209 \\
35 & 27.0385 & 28.3981 & 29.7250 & 33.4588 \\
45 & 26.3121 & 27.0427 & 28.4824 & 29.0612 \\
55 & 25.6314 & 26.3359 & 27.5676 & 28.8872 \\
\hline
\end{tabular}


In order to analyze the proposed method in performing effectively noise reduction, the Lena image is chosen as a standard test image. We first add white noise to the Lena image with standard variance $\sigma=25,35,45,55$. A non-fractional differential based image enhancement method (Tang, et al. [14]) and another two fractional based methods (Tian's method [13], FCD-1 method [9]) are used to perform comparisons with our proposed method. The PSNR (peak signal-to-noise ratio) measure is adopted to evaluate the quality of the enhanced image, which is defined as follows:

$$
\begin{gathered}
P S N R=10 \cdot \log _{10}\left(\frac{M A X_{f}^{2}}{M S E}\right) M A X_{f} \\
M S E=\frac{1}{M \times N} \sum_{i=0}^{M-1} \sum_{j=0}^{N-1}[f(i, j)-g(i, j)]^{2}
\end{gathered}
$$

where MSE is the mean squared error of original image and enhanced image, $f$ is a $M \times N$ input image and $g$ represents the enhanced image that is of the same size with $f$, and PSNR can be defined by the MSE and $M A X_{f}$, which is the maximum possible pixel value of the image.

It can be clearly seen from Table 1 that the fractional operator methods have a better performance than the non-fractional differential based method, because they can enhance the edge information and preserve the texture details within the smooth areas nonlinearly in accord with the original nonlinear characteristic of image signal. In addition, the proposed method based on variable-order fractional differential operator with mask size of $5 * 5$ obtains a high PSNR relative to the last two methods (FCD-1 and Tian's method).

\section{Discussion}

According to the experimental results and analysis above, the FPDF-2 discrete formula outperforms the other four different discretization formulas. Both of the two evaluation standards in Figure 4 show that the FPDF-3 method has a worse performance compared with FPDF-2. The reason is that the first few coefficients will increase along with the rising discrete order of the fractional differential derivative; in other words, there will be a higher peak value at the central point of the mask by using FPDF-3 than FPDF-2. When this mask is used to enhance the detail information of the image, it will enlarge the noise existed in smooth areas due to the high peak values in the mask, just as Figure 4 shows that the contrast for FPDF-3 starts to turn around when fractional order $v$ is around 0.8 . Therefore, we can conclude that a discrete order beyond a certain maximum threshold will have a negative effect on the performance of image enhancement. However, how to determine the specific maximum threshold for the discrete order is still a problem to be investigated in future work. In addition, the fractional differential order is also a key factor that determines the final quality of the enhanced image, for the reason that its value has a great influence on the size of coefficients of the fractional differential mask, the same as the discrete order, whereas an extremely high fractional order will lead to bad quality of the enhanced image. Hence, variable-order fractional differential operators (or masks) combined with detailed image information may provide synergistic means to solve this problem. 


\section{Conclusion}

Fractional differential based methods have experienced a rapid development in the recent years, and fractional modeling has received wide attention from different research areas. In this paper, a variableorder fractional differential operator based approach is proposed for image enhancement. Moreover, the influence of five different discrete methods to image enhancement has been studied. The experiements show that the FBDF-p discrete method has a good performance for preserving the texture information while filtering the image, and the optimized mask can greatly reduce the image noise in smooth area resulted from the enhacement with uniform-order operator.

\section{Acknowledgments}

This work was supported by the National Science-technology Support Plan (2014BAI17B02) and Fundamental Research Funds for the Central Universities under grant N140402003. Partial support was provided by NIH grant (1U01HL116323) while the first author was visiting Brown University.

\section{References}

[1] M. Adhi and J.S. Duker, Optical coherence tomography--current and future applications, Current Opinion in Ophthalmology 24 (2013), 213-221.

[2] E. Real, E. Alma and P. Alejandro, Optical coherence tomography assessment of vessel wall degradation in thoracic aortic aneurysms, Journal of Biomedical Optics 18 (2013), 126003.

[3] N. Padmasini, K.S. Abbirame and S.M. Yacin, Speckle noise reduction in spectral domain optical coherence tomography retinal images using anisotropic diffusion filtering, IEEE International Conference on Science Engineering and Management Research (ICSEMR) 12 (2014), 1-5.

[4] M.R. Avanaki, M.J. Marques and A. Bradu, A new algorithm for speckle reduction of optical coherence tomography images, Proceedings of SPIE in Optical Coherence Tomography and Coherence Domain Optical Methods in Biomedicine XVIII 8934 (2014), 893437.

[5] H.M. Salinas and D.C. Fernández, Comparison of PDE-based nonlinear diffusion approaches for image enhancement and denoising in optical coherence tomography, IEEE Transactions on Medical Imaging 26 (2007), 761-771.

[6] M. Janev, S. Pilipović and T. Atanacković, Fully fractional anisotropic diffusion for image denoising, Mathematical and Computer Modelling 54 (2011), 729-741.

[7] P. Gatto and J.S. Hesthaven, Numerical approximation of the fractional Laplacian via hp-finite elements, with an application to image denoising, Journal of Scientific Computing 10 (2014), 1-22.

[8] Y.F. Pu, J.L. Zhou and X. Yuan, Fractional differential mask: A fractional differential-based approach for multiscale texture enhancement, IEEE Transactions on Image Processing 19 (2010), 491-511.

[9] Q. Yu, F. Liu and I. Turner, The use of a Riesz fractional differential-based approach for texture enhancement in image processing, Australian and New Zealand Industrial and Applied Mathematics Journal 54 (2013), C590-C607.

[10] F. Hu, S. Si, and H.S. Wong, An adaptive approach for texture enhancement based on a fractional differential operator with non-integer step and order, Neurocomputing 12 (2015), 295-306.

[11] C. Li and F. Zeng, Finite difference methods for fractional differential equations, International Journal of Bifurcation and Chaos 22 (2012), 1230014.

[12] C. Li and F. Zeng, The finite difference methods for fractional ordinary differential equations, Numerical Functional Analysis and Optimization 34 (2013), 149-179.

[13] W. Tian, H. Zhou and W. Deng, A class of second order difference approximations for solving space fractional diffusion equations, Mathematics of Computation, 2015. (in press)

[14]J.R. Tang and N.A.M. Isa, Adaptive image enhancement based on bi-histogram equalization with a clipping limit, Computers \& Electrical Engineering 40 (2014), 86-103. 